\title{
Ultra-small dye-doped silica nanoparticles via modified sol-gel technique
}

\author{
R. Riccò • S. Nizzero • E. Penna • A. Meneghello • \\ E. Cretaio • F. Enrichi $(\mathbb{D}$
}

Received: 12 December 2017 / Accepted: 13 April 2018 / Published online: 25 April 2018

(C) The Author(s) 2018

\begin{abstract}
In modern biosensing and imaging, fluorescence-based methods constitute the most diffused approach to achieve optimal detection of analytes, both in solution and on the single-particle level. Despite the huge progresses made in recent decades in the development of plasmonic biosensors and label-free sensing techniques, fluorescent molecules remain the most commonly used contrast agents to date for commercial imaging and detection methods. However, they exhibit low stability, can be difficult to functionalise, and often result in a low signal-to-noise ratio. Thus, embedding fluorescent probes into robust and bio-compatible materials, such as silica nanoparticles, can substantially enhance the detection limit and dramatically increase
\end{abstract}

R. Riccò and S. Nizzero contributed equally to this work.

\section{R. Riccò $(\bowtie)$}

Institute of Physical and Theoretical Chemistry, Graz University of Technology, Stremayrgasse 9, 8010 Graz, Austria

e-mail: raffaele.ricco@tugraz.at

S. Nizzero

Department of Physics, University of Padova, via Marzolo 8, 35131 Padua, Italy

E. Penna

Department of Biomedical Sciences, University of Padova, Via Ugo Bassi 58/B, 35131 Padua, Italy

\section{A. Meneghello}

CRO National Cancer Institute, Via Franco Gallini 2,

33081 Aviano, PN, Italy the sensitivity. In this work, ultra-small fluorescent silica nanoparticles (NPs) for optical biosensing applications were doped with a fluorescent dye, using simple waterbased sol-gel approaches based on the classical Stöber procedure. By systematically modulating reaction parameters, controllable size tuning of particle diameters as low as $10 \mathrm{~nm}$ was achieved. Particles morphology and optical response were evaluated showing a possible single-molecule behaviour, without employing microemulsion methods to achieve similar results.

Keywords Silica nanoparticles - Dye doping · Luminescence $\cdot$ Biosensing $\cdot$ Bioimaging applications

\author{
E. Cretaio \\ Department of Molecular Sciences and Nanosystems, University \\ of Venice Ca' Foscari, Via Torino 155, 30172 Mestre, VE, Italy \\ F. Enrichi $(\square)$ \\ Division of Materials Science, Department of Engineering \\ Sciences and Mathematics, Luleå University of Technology, \\ 97187 Luleå, Sweden \\ e-mail: francesco.enrichi@1tu.se \\ F. Enrichi \\ Museo Storico della Fisica e Centro Studi e Ricerche Enrico \\ Fermi, Piazza del Viminale 1, 00184 Roma, Italy
}




\section{Introduction}

A fundamental requirement for highly responsive optics-based biosensors is to have enough signal-tonoise ratio (SNR) to reveal the presence of analytes at very low concentrations (Banica 2012). Huge efforts were spent along the years to develop stable, efficient, highly emitting optical labels with optimal spectral properties, with cost-effective production procedures easy to scale up for commercial production (Syahir et al. 2015). Among these properties, the most important ones required for optical labels for biosensing technologies are high biocompatibility, high SNR, and low photodegradation. In an initial effort of developing alternative strategies for high sensitivity detection using time-delayed analysis, the use of Europium-doped silica NPs was found to provide enough long-living signal (in the order of milliseconds) from the emission of rare earth ion with respect to the short-living self-fluorescence (in the order of ns) of the biological system (Enrichi et al. 2008; Canton et al. 2011). Other rare earth metals, and luminescent silica NPs in place of directly link emitting molecules, were also explored (Enrichi 2008). One of the advantages of dye-doped nanoparticles over other luminescent nanoparticles like quantum dots or rare earth-doped NPs is to provide the same optical properties of the incorporated dyes, which are usually commercial dyes. Therefore, they are perfectly matched to the typical spectral response of standard laboratory instruments fitted to commercial dyes, but at the same time they can provide much higher performances. Indeed, the use of dye-doped silica NPs was demonstrated to improve significantly the detection limit of a microarray device (Enrichi et al. 2010), permitting the implementation of this fluorescent nanosystem in the in vitro DNA microarray detection of human papilloma virus (HPV) single-strand sequences (Riccò et al. 2011). All these studies suggest the use of $d y$ - $d$ oped silicon oxide nanoparticles (ddSiONPs) as a promising strategy to overcome the drawbacks of fluorescent molecule labels. In fact, the surface of silica NPs can be easily modified to link any suitable bio-probe ( $\mathrm{Li}$ et al. 2012). Furthermore, by incorporating dyes into silica spheres, a higher concentration of fluorescence emitters can be obtained within a single optical label, which yields to improve SNR, and the molecules inside the silica network are protected from the environment, reducing photobleaching (Canton et al. 2011). Small size is a key requirement for this new class of fluorescent labels, to ensure the system can be easily substituted to traditional single-molecule labels, to avoid photobleaching and photoblinking, and to improve chemical stability and bio-conjugation efficiency. The use of silica particles has also been shown very promising for clinical use, thanks to its high biocompatibility (Asefa and Zhimin 2012). The $<10$-nm size is particularly important for in vivo applications, where the size is a major determinant in biodistribution and pharmacokinetic.

Low-size particles are often obtained using microemulsion methods (Esquena et al. 1997; Lade et al. 2000; Bagwe et al. 2004; Finnie et al. 2007). Several groups around the world have focused on developing methods in this field to fabricate ultra-small dye-doped silica particles with regular pore sizes. For example, Ma et al. systematically investigated the fabrication of both dense and mesoporous sub-10-nm silica particles, using CTAB surfactant and PEG-silane (Ma et al. 2012a, b). Recently, Quan et al., fabricated sub-10$\mathrm{nm}$ fluorescent particles in alkaline buffer and in presence of cyclohexane to produce a biphasic system (Quan et al. 2017).

However, these techniques may present environmental and technical drawbacks hampering their scalability and making the production process less attractive for commercial applications. For example, the reaction vessels must be able to withstand organic compounds (solvents, surfactants) used for the inevitable water-in-oil emulsion, and base catalysts; some organic solvents can pose an environmental and health hazard; the surfactant must be chosen to precisely generate defined micelles; the doping agent must be hydrophilic to enter the inner aqueous micellar environment; and the work-up requires breaking of micelles and extensive washing to remove surfactant, solvents, oligomers, and other byproducts.

To the purpose of biosensing, porosity is not a key factor, whether scalability is crucial to allow commercial applications. A classic and easily scalable Stöber method (Stöber et al. 1968) can provide mono-dispersed and well-defined particles, but only in the 100-1000-nm range, where "nano" systems become "micro" systems (Alemán et al. 2007). A notable reduction in NP size has been recently achieved (Canton et al. 2011) with a timeand cost-efficient technique following a modified Stöber basic environment sol-gel procedure, using tetraethyl orthosilicate (TEOS) and aminopropyl triethoxysilane (APTES) in ethanol environment, AlexaFluor ${ }^{\circledR}$ dyes as doping agents dissolved in dimethyl sulfoxide (DMSO), 
and ammonia solution as catalyst. NPs in the 15-70-nm range were easily produced, and the effect of dye loading and APTES amount in the resulting diameters was thoroughly studied (Canton et al. 2011).

In the present work, we will provide guidelines to synthesise nanosized luminescent silica labels for fluorescence-based biological sensing applications, showing a simple, efficient, and sustainable protocol for the synthesis and systematic tuning of ddSiONPs in the ultra-small scale range $(<30 \mathrm{~nm})$, by modification of the classic base-catalysed, sol-gel hydrolysis, and condensation of TEOS (Brinker and Scherer 1990), using fluorescein as probe and, importantly, with analogous results that were obtained with microemulsion methods.

\section{Experimental}

Materials and methods

Tetraethyl orthosilicate (TEOS 98\%), 3-aminopropyl triethoxysilane (APTES), 5(6)-carboxyfluorescein (FCOOH), dicyclohexylcarbodiimide (DCC), 4- $(N, N-$ dimethylamino)-pyridine (DMAP), and ammonia solution ( 25 or $30 \%$ aqueous) were purchased from Sigma Aldrich.

Acetone, ethanol (EtOH), dimethyl sulfoxide (DMSO), ethyl acetate (EtOAc), and tetrahydrofuran (THF) were used without further purification.

SEM measurements were performed with a TESCAN Vega TS 5130 LM working at $30 \mathrm{kV}$. Highperformance FESEM investigations were conducted on a dual-beam FEI Nova 600i instrument, with a semi-inlens cold cathode field emission scanning electron microscope source. The micrographs were taken at $5-\mathrm{kV}$ accelerating voltage, using in-lens detector in pure secondary electron signal mode. In all cases, a thin layer of gold was sputtered onto samples.

Atomic force microscopy (AFM) maps were produced using a NT-MDT Ntegra instrument in a semicontact mode with gold-coated Si cantilevers Etalon or NSG01, 03, 10, 30 types. A typical measure scans a $75 \times 75$ to $5 \times 5 \mu^{2}$ area, with a scanning frequency between 0.2 and $0.5 \mathrm{~Hz}$, depending on the surface.

DLS measurements were performed with a Malvern Instruments Zetasizer.
Synthesis of dye adduct fluorescein APTES

In a round-bottom, two-neck, 250-mL Schlenck-lined flask, under magnetic stirring and nitrogen stream, $564 \mathrm{mg}(1.5 \mathrm{mmol})$ of FCOOH is dissolved in $30 \mathrm{~mL}$ of anhydrous acetone. Two solutions of $402 \mathrm{mg}$ ( $2 \mathrm{mmol}$ ) of DCC in $10 \mathrm{~mL}$ of acetone and $488 \mathrm{mg}$ ( $4 \mathrm{mmol}$ ) of DMAP in $10 \mathrm{~mL}$ of acetone were added and the mixture was stirred for $15 \mathrm{~min}$. Subsequently, $432 \mathrm{mg}$ ( $463 \mu \mathrm{L}, 2 \mathrm{mmol})$ of APTES was injected and the stirring continued for another $90 \mathrm{~min}$.

After solvent removal, the residue was taken with EtOAc, washed $2 \times 100 \mathrm{~mL}$ with citric acid (1\% aqueous solution) and $1 \times 100 \mathrm{~mL}$ with brine. Organic phase was dried over $\mathrm{MgSO}_{4}$ and solvent removed, leaving FCPTES as yellow solid (800 mg, 92\%). TLC ( $\mathrm{SiO} 2$, EtOAc/EtOH 8:2): $R_{\mathrm{f}}=0.32$. 1H-NMR $(\mathrm{CDCl} 3$, $300 \mathrm{MHz}): 10.17 \mathrm{ppm}(\mathrm{s}, 1 \mathrm{H}, \mathrm{COOH})$, $7.19 \div 8.45 \mathrm{ppm}(\mathrm{m}, 7 \mathrm{H}, \mathrm{CH}$ Ar xanthene $+\mathrm{NH})$, $6.50 \div 6.75 \mathrm{ppm}(\mathrm{m}, 3 \mathrm{H}, \mathrm{CH}$ phenyl), $4.03 \mathrm{ppm}(\mathrm{t}, 2 \mathrm{H}$, $\left.\mathrm{N}-\mathrm{CH}_{2}, J=7.1 \mathrm{~Hz}\right), 1.58 \div 1.72 \mathrm{ppm}\left(\mathrm{m}, 10 \mathrm{H}, \mathrm{CH}_{2}\right.$ propyl and $\left.\mathrm{Si}-\mathrm{O}-\mathrm{CH}_{2}\right), 1.01 \div 1.25\left(\mathrm{~m}, 9 \mathrm{H}, \mathrm{CH}_{3}\right)$. FTIR (KBr) 3479, 3329, 3061, 2929, 2851, 1749, 1629, 1510, 1384, 1244, 1184, 1111, 844, and $661 \mathrm{~cm}^{-1}$. UV-Vis (EtOH, $0.4 \mathrm{mM}$ ): $\lambda_{\max }=454$ and $484 \mathrm{~nm}$.

General methods for low-size $(<30 \mathrm{~nm})$ silica nanoparticles synthesis

In a plastic, conical bottom, 50-mL vial provided with magnetic stirrer, ethanol, $18.3 \mathrm{M} \Omega$ deionised water, TEOS, and ammonia solution were added and mixed for 18-24 h. After that, purification method was followed as explained in the following section. For dye-doped particles, the fluorophore is previously dispersed in $2 \mathrm{~mL}$ of DMSO or ethanol (see synthesis details in Table 1). Fluorescein@ $\mathrm{SiO}_{2} \mathrm{NPs}$ were prepared using $10 \mathrm{mM}$ ethanol or DMSO solutions of FCPTES adducts.

General method for low-size $(<30 \mathrm{~nm})$ silica nanoparticles purification

Amicon-15 ultrafiltration 50-mL vials (Millipore), with a 20 -to $100-\mathrm{kDa}$ cut-off, were used. The reaction mixture was diluted with the same volume of $18.3 \mathrm{M} \Omega$ deionised water, then poured in $10-\mathrm{mL}$ portions in the upper part of the vial; every portion is centrifuged at $5000 \mathrm{rpm}$ for $20 \mathrm{~min}$, the underlying filtrate discarded, 
and the above concentrate dispersion collected. After completion, all the fractions were put together, diluted to $10 \mathrm{~mL}$ with $18.3 \mathrm{M} \Omega$ deionised water, put into an Amicon-15 vials (Millipore) with $50-\mathrm{kDa}$ cut-off, and centrifuged at $5000 \mathrm{rpm}$ for $30 \mathrm{~min}$, with $2 \times 25 \mathrm{~mL}$ water rinsing. Before the centrifugation step, vial was vigorously shaken to prevent filter clogging. The obtained concentrate solution is collected, diluted to $2 \mathrm{~mL}$ with water or buffer solution and stored at $4{ }^{\circ} \mathrm{C}$.

\section{Results and discussion}

The integration of fluorescein isothiocyanate (FITC) within inorganic silica frameworks is usually conducted by the addition APTES to FITC (Enrichi et al. 2009a, 2010; Riccò et al. 2011). Here, we explored an alternative pathway in order to extend this approach to other fluorescent molecules lacking the iso(thio)cyanate moiety. In this regard, we used a traditional amide formation method (Neises and Steglich 1978) for the synthesis of siloxane-derivatized dyes through the formation of an activated ester, using APTES and 5(6)-carboxyfluorescein, as depicted in Fig. 1.

This approach brings several advantages: fast reaction at room temperature thanks to $N, N$ dimethylaminopyridine (DMAP) acceleration, strong water harvesting by dicyclohexylcarbodiimide (DCC) to avoid siloxane hydrolysis, selective functionalization on the desired moiety, easy execution, high yield (>
90\%), and environmental compatibility. The sol-gel silica nanoparticle synthesis has been performed according to the procedure pioneered by Stöber, Bogush, and van Blaaderen (Stöber et al. 1968; Bogush et al. 1988; van Blaaderen and Vrij 1992). In their work, TEOS in an ethanol solution along with variable amounts of ammonia and water underwent hydrolysis and condensation phenomena to produce small silicon dioxide spheres with different diameters, usually bigger than $30 \mathrm{~nm}$.

In the first set of experiments, the chosen co-solvent (DMSO) was added to solubilise the dye in the reaction medium. Previous studies demonstrated the formation of two distinct families of nanoparticles with 400- and 750-nm diameter, a phenomenon credited to the DMSO presence. However, according to scanning near-field optical microscopy (SNOM) measurements, only 750$\mathrm{nm}$ particles were found to contain dye molecules (Enrichi et al. 2009b). Here, the experiments were conducted varying the volumes of DMSO solution (Table 1, samples D14F2, D7F0, and D3F0). The typical molar ratio of sol-gel solutions TEOS/EtOH/ $\mathrm{H}_{2} \mathrm{O} / \mathrm{NH}_{3}$ was 1:86:33.2:2.4, corresponding to a mixture of $900 \mu \mathrm{L}$ TEOS, $20 \mathrm{ml} \mathrm{EtOH}, 2.5 \mathrm{ml} \mathrm{H}_{2} \mathrm{O}$, and $800 \mu \mathrm{L}$ of $25 \%$ aqueous ammonia solution. Control samples were generated omitting DMSO (Table 1, samples D0F2 and D0F5).

In the second set of experiments, the concentration of reactants in solvent $(\mathrm{EtOH})$ was modulated by varying the total amount of EtOH. This set (Table 1, samples E86 to E258) was produced fixing the TEOS/ $\mathrm{H}_{2} \mathrm{O} / \mathrm{NH}_{3}$
Table 1 Molar ratios of reaction species $($ TEOS $=1$ ) with diameters obtained from dimensional measurements

D7F0: $\mathrm{PDI}=0.24 ; \mathrm{D} 3 \mathrm{~F} 0: \mathrm{PDI}=$ 0.14

${ }^{\mathrm{a}}$ Additional $\mathrm{H}_{2} \mathrm{O}=31.4$

${ }^{\mathrm{b}} \mathrm{AFM}$

${ }^{\mathrm{c}} \mathrm{SEM}$

${ }^{\mathrm{d}}$ DLS

\begin{tabular}{lllllll}
\hline Sample & EtOH & $\mathrm{H}_{2} \mathrm{O}$ & $\mathrm{NH}_{3}$ & DMSO & FCPTES $\left(\times 10^{-4}\right)$ & Avg. $\varnothing(\mathrm{nm})$ \\
\hline D0F2 & 86 & 33.2 & 2.4 & & 2 & $32 \pm 3^{\mathrm{b}}$ \\
D0F5 & 86 & 33.2 & 2.4 & & 5 & $35 \pm 3^{\mathrm{b}}$ \\
D14F2 & 86 & 33.2 & 2.4 & 14.2 & 2 & $3 \pm 1^{\mathrm{b}}$ \\
D7F0 & 86 & 33.2 & 2.4 & 7.1 & & $5 \pm 1^{\mathrm{b}}\left(14 \pm 7^{\mathrm{d}}\right)$ \\
D3F0 & 86 & 33.2 & 2.4 & 3.5 & & $15 \pm 3^{\mathrm{b}}\left(20 \pm 7^{\mathrm{d}}\right)$ \\
E86 & 86 & 5.3 & 2.4 & 14.2 & 2 & $9 \pm 2^{\mathrm{c}}$ \\
E129 & 129 & 5.3 & 2.4 & 14.2 & 2 & $13 \pm 4^{\mathrm{c}}$ \\
E172 & 172 & 5.3 & 2.4 & 14.2 & 2 & $18 \pm 5^{\mathrm{c}}$ \\
E215 & 215 & 5.3 & 2.4 & 14.2 & 2 & $8 \pm 2^{\mathrm{c}}$ \\
E258 & 258 & 5.3 & 2.4 & 14.2 & 2 & $7 \pm 2^{\mathrm{c}}$ \\
W2N1 & 200 & $2.2\left(33.6^{\mathrm{a}}\right)$ & 1 & & 1 & $12 \pm 4^{\mathrm{c}}$ \\
W4N2 & 200 & $4.4\left(35.8^{\mathrm{a}}\right)$ & 2 & & 1 & $23 \pm 5^{\mathrm{c}}$ \\
W8N4 & 200 & $8.8\left(40.2^{\mathrm{a}}\right)$ & 4 & & 1 & $55 \pm 8^{\mathrm{c}}$ \\
W17N8 & 200 & $17.6\left(49.0^{\mathrm{a}}\right)$ & 8 & & 1 & $200 \pm 20^{\mathrm{c}}$ \\
W26N12 & 200 & $26.3\left(57.7^{\mathrm{a}}\right)$ & 12 & & 1 & $250 \pm 25^{\mathrm{c}}$ \\
\hline
\end{tabular}




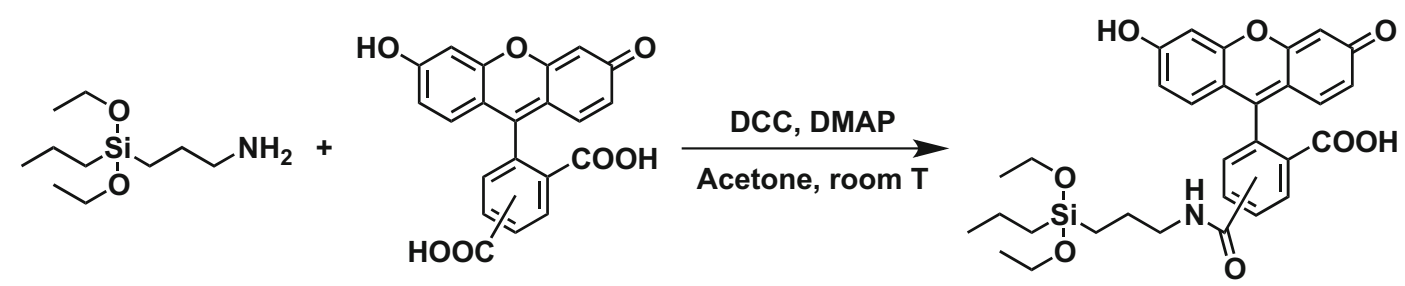

Fig. 1 Reaction scheme for the synthesis of FCPTES. APTES reacts with 5(6) carboxyfluorescein to form FCPTES. Room $T=24{ }^{\circ} \mathrm{C}$

/DMSO/FCPTES in the 1:5.3:2.4:14.2:2 $\times 10^{-4} \mathrm{M}$ ratio, corresponding to $450 \mu \mathrm{L}$ TEOS, $300 \mu \mathrm{L}$ of $30 \%$ aqueous ammonia solution, and $2 \mathrm{~mL}$ of a FCPTES solution in DMSO $(1 \mathrm{mg} / \mathrm{mL})$. Ethanol was tuned from 86 to 258 equivalents with respect to TEOS, which resulted in a variable volume from 10 to $30 \mathrm{~mL}$. In the third set of experiments, the investigation focused on the proportion between reactants and ammonia. In this final set (Table 1, samples W1N1 to W26N12), DMSO was not added, and a fixed TEOS/EtOH/ $\mathrm{H}_{2} \mathrm{O} / \mathrm{FCPTES}$ ratio 1:200:31.4:8.5 $\times 10^{-5}$ was used, corresponding to $450 \mu \mathrm{L}$ TEOS, $23 \mathrm{~mL}$ EtOH, $1.125 \mathrm{~mL} \mathrm{H}_{2} \mathrm{O}$, and $0.1 \mathrm{mg}$ of FCPTES. In this case, the ammonia molar amount rose from 33 to 58 equivalents, thus from $125 \mu \mathrm{L}$ to $1.5 \mathrm{~mL}$ of $30 \%$ aqueous ammonia solution. The amount of water present as the diluent within the ammonia solution was included in the calculation of the total water amount reported in Table 1.

Nanoparticles with mean diameter of $32 \pm 3 \mathrm{~nm}$ were measured for sample D0F2, whereas sample D0F5 provided particles with $35 \pm 3 \mathrm{~nm}$ average diameter: the slight difference was attributed to the bigger amount of FCPTES used, that is, likely to have caused a slight rise in the average diameter due to the additional silica precursor added. When DMSO (2 $\mathrm{mL})$ was added to the same solution, a significant diameter decrease was observed. The obtained nanoparticles passed from $32 \pm$ $3 \mathrm{~nm}$ (sample D0F2) to $3 \pm 1 \mathrm{~nm}$ (sample D14F2), a tenfold shrinking currently unreported in literature. Samples D7F0 and D3F0 were produced without dye doping, to investigate the influence of DMSO concentration on the mean diameter of the obtained nanoparticles. The dimensional trend with lowering DMSO concentration went from $5 \pm 1 \mathrm{~nm}$ for D7F0 to $15 \pm 3 \mathrm{~nm}$ for D3F0. AFM measurements were compared to dynamic light scattering (DLS) measurements: size trend as a function of DMSO concentration was consistent between the two methods. However, DLS measurements give an estimate of the hydrodynamic radius, whereas
AFM measures the geometrical radius. Thus, DLS estimates of radii were systematically slightly higher as compared to those in AFM.

The second series (E86-E258) has been produced removing the initial water and increasing the $\mathrm{EtOH}$ amount, to investigate the influence in the diameter. Sample size was investigated using AFM (Fig. 2 left), field emission scanning electron microscopy (FESEM, Fig. 2 right), dynamic light scattering (DLS, Fig. 2 center), and transmission electron microscopy (TEM). The obtained particles had actually an irregular distribution and seemed not to be heavily affected by the dilution. For example, microscopy measurements on sample E258 yielded an average diameter of $7 \pm 2 \mathrm{~nm}$. All the samples of the same set range between 7 and $18 \mathrm{~nm}$, without a significant trend. From this investigation, it is clear that ethanol has a minor role in this range of size values. Instead, in this low diameter regime, the size is mainly determined by DMSO. Furthermore, by doubling the solvent molar ratio toward TEOS and drastically reducing the water amount, small particles under $20 \mathrm{~nm}$ were produced in presence of DMSO co-solvent. The mechanism behind this behaviour is likely due to the role of the polar aprotic solvent DMSO. It is well known that such class of solvents, albeit not hydrogen bonding, stabilises the reactants with respect to the activated complex, slowing down the reaction and inducing the formation of smaller nuclei (Brinker and Scherer 1990).

To achieve the goal of fine-tuning nanoparticle diameters in the range of ultra-small particles, the third set W2N1-W26N12 focused on using various ammonia concentrations. DMSO was removed and the ammonia molar ratio was tuned between 1 and 12, using a fixed TEOS/EtOH $/ \mathrm{H}_{2} \mathrm{O}$ ratio of $1: 200: 31.4$. FCPTES was kept at a $0.01 \%$ mol with respect to TEOS in all cases. It is noteworthy that proportions between ammonia, water, solvent, and other species are fundamental when producing silica nanoparticles by means of 

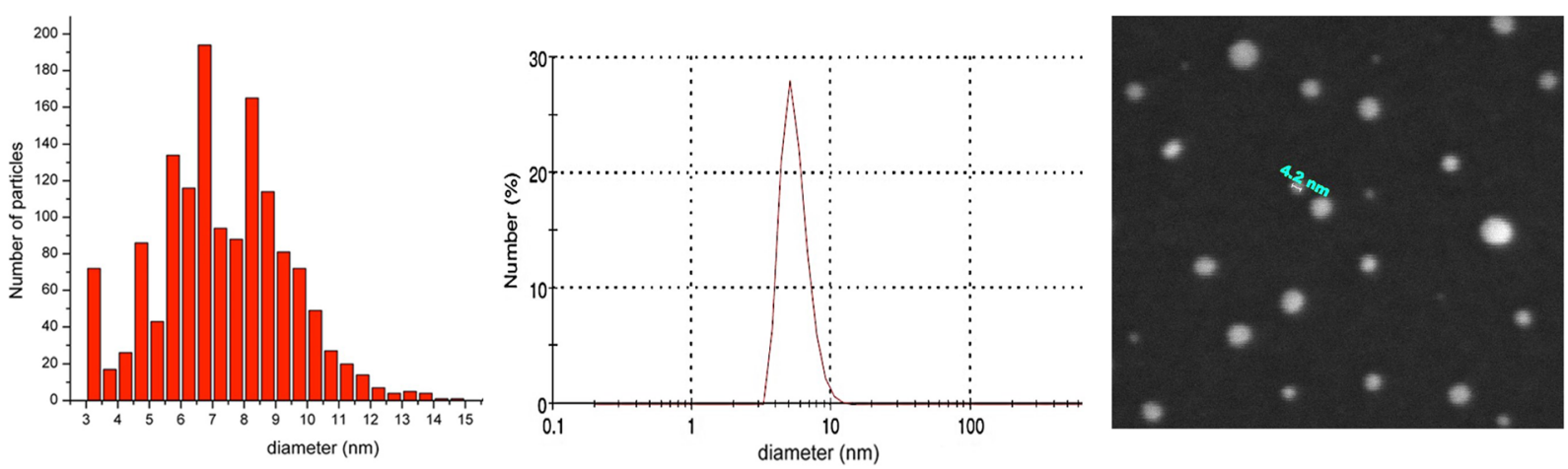

Fig. 2 Size characterisation of samples E258 by AFM (left) and DLS (center). On the right, FESEM image of the same sample

microemulsion systems (Bagwe et al. 2004). As seen in Fig. 3, nanoparticle dimensions were directly proportional to the amount of ammonia used, which can be explained based on sol-gel techniques established mechanism (Brinker and Scherer 1990.

The dimensional control was well maintained and it was possible to reach very low particle size by simply tuning the amount of base catalyst or co-solvent (Fig. 4). For samples W2N1 and W4N2, 125 and $250 \mu \mathrm{L}$ of $30 \%$ aqueous ammonia solution were used, respectively. The aqueous molar ratios became 33 and 35 and the ammonia molar ratios were between 1 and 2 with respect to TEOS. Consequently, an equimolar amount of TEOS and ammonia was sufficient to produce very low sized silica nanoparticles, around $10 \mathrm{~nm}$, in ethanol environment with nearly $15 \%$ of water. A twofold amount of ammonia was able to double the diameter without particular issues, thus providing a linear dependence between catalyst and dimension in the range 5-60 nm (samples W2N1, W4N2, and W8N4).
Fig. 3 Micrographs for samples W2N1 (a), W4N2 (b), W8N4 (c), and W26N12 (d).

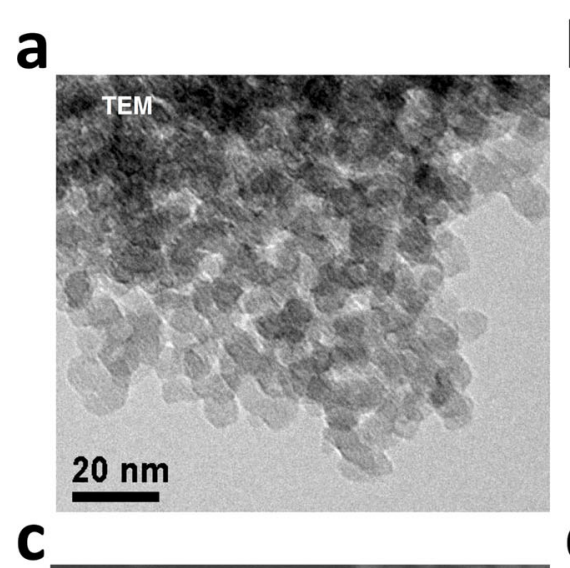

b

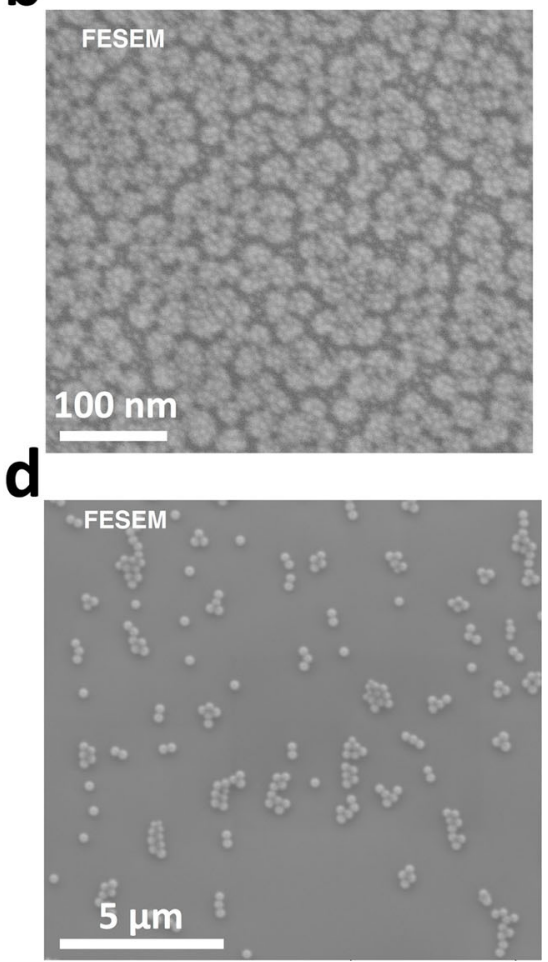



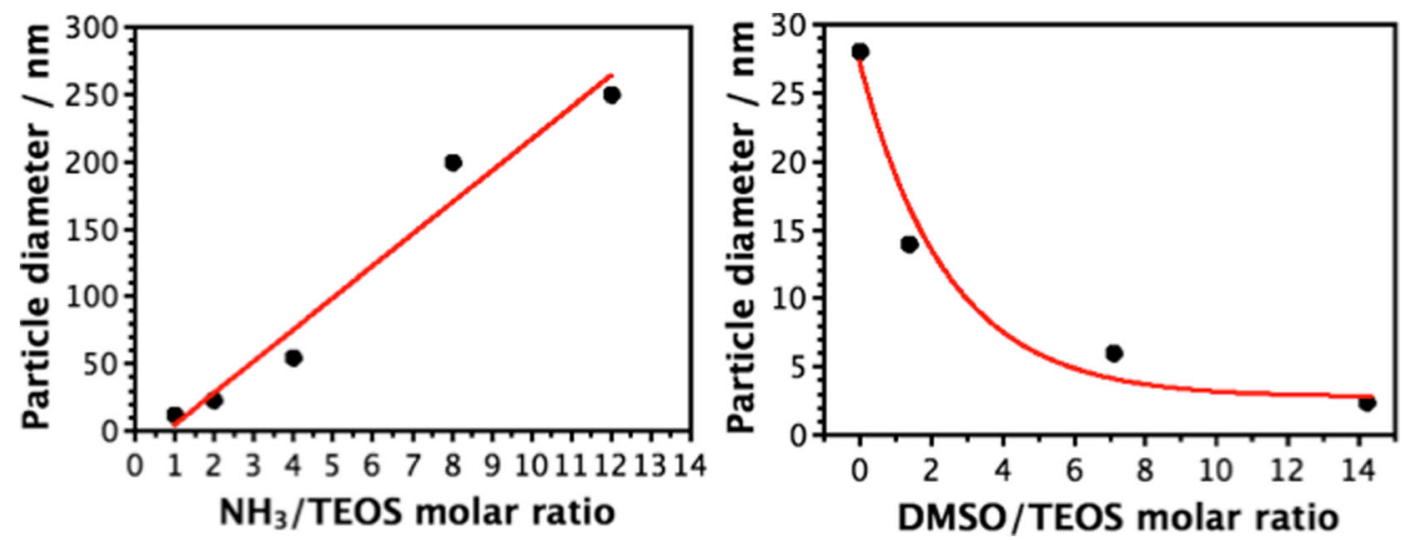

Fig. 4 Dependence of particle size from amount of base catalyst $\left(\mathrm{NH}_{3}\right.$ solution) (a) and amount of co-solvent (DMSO) (b)

Optical characterisation of the prepared nanoparticles confirmed the dye loading efficiency. Fluorescein was chosen as a widespread, cost-effective, and well characterised dye. In Fig. 5 left, the normalised optical absorption (black), photoluminescence (PL) excitation (red), and emission (blue) spectra of the $35 \pm 3 \mathrm{~nm}$ D0F5 nanoparticles sample are reported. The clear difference between the PL excitation and the absorption spectra is attributed to the higher dye concentration in the synthesis and to the bigger particle size, allowing for the incorporation of dye molecules in close proximity, favouring energy transfer phenomena among them, and the probability of reabsorption or quenching phenomena. In Fig. 5 right, the normalised optical absorption (black), PL excitation (red), and emission (blue) spectra of the $3 \pm 1 \mathrm{~nm}$ D14F2 nanoparticles are reported. These are in perfect agreement with the optical properties of a free dye, indicating incorporation of fluorescein in the nanoparticle without modification of its molecular structure (Lakowicz 2006). Moreover, the overlap between the photoluminescence excitation curve and absorption spectrum curve is complete and is indicative of no re-absorption or concentration-quenching processes. Thus, no deformation of the photoluminescence spectral shape both in the excitation and in the emission occurred. Given the reduced size of this sample and being a fluorescein molecule approximately $0.6 \times 0.9 \times 0.9 \mathrm{~nm}$ wide, it is reasonable to imagine either a single-molecule occupancy per particle or a single-molecule behaviour within a multiple-occupied particle, similarly to what has been previously demonstrated by Douhal and co-workers with another cyanine-based dye (Cohen et al. 2012). Future studies, beyond the scope of this work, would be

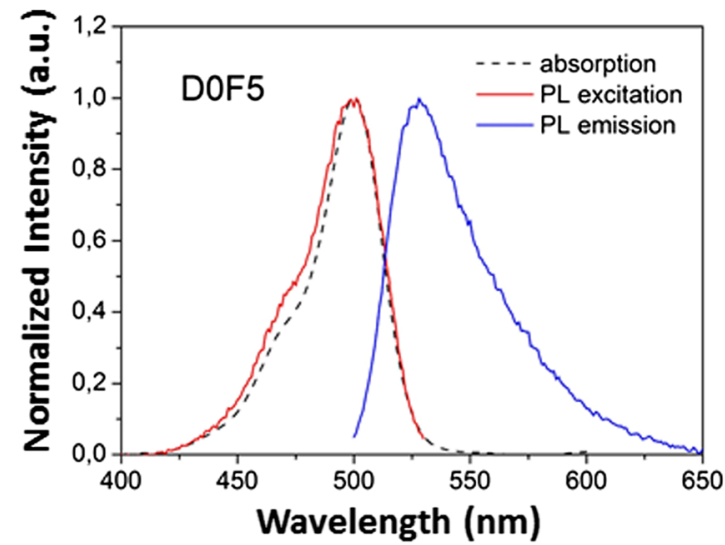

Fig. 5 Absorption, PL excitation, and PL emission for the $35 \pm$ $3 \mathrm{~nm}$ D0F5 (left) and $3 \pm 1 \mathrm{~nm}$ D14F2 (right) nanoparticles. The PL excitation curve (red) was obtained monitoring emission

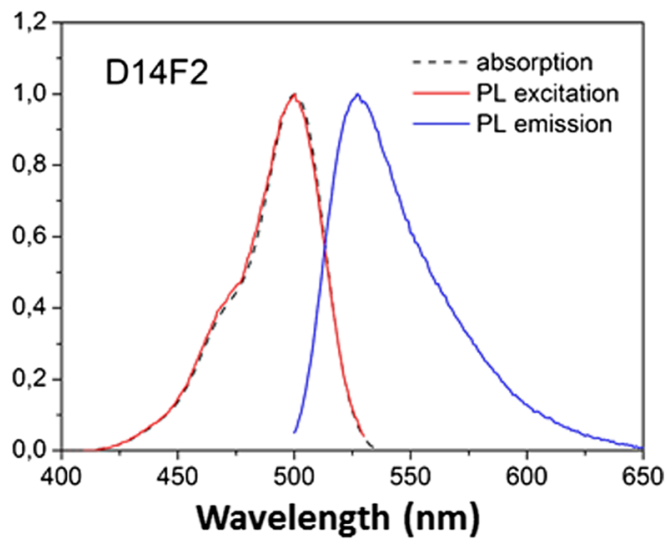

wavelength $\lambda_{\mathrm{em}}=530 \mathrm{~nm}$. The PL emission curve (blue) was obtained under excitation wavelength $\lambda_{\mathrm{ex}}=470 \mathrm{~nm}$ 
necessary to assess the number of molecules per particle and to investigate the applicability of such systems to commercial biosensors.

\section{Conclusions}

In this work, fluorescein-doped silica nanoparticles with a diameter down to $10 \mathrm{~nm}$ or less could be easily produced in an environmentally friend fashion, by a simple modification of the classical Stöber technique without the needs of microemulsion systems, avoiding the use of organic solvents and surfactants. This was achieved by adapting the amounts of ethanol and ammonia solution, reducing the amount of water. DMSO presence was found to induce the formation of very lowsized nanoparticles, but a single solvent would be preferred for the scalability of the production process. The technique of choice comprised a mixture of $\mathrm{TEOS} / \mathrm{EtOH} / \mathrm{NH}_{3} / \mathrm{H}_{2} \mathrm{O}$ in the molar ratio $1: 200: 1 \div 2: 35$, and can produce nanoparticles in the 10 - to 20 -nm range, as seen by AFM, DLS, and FESEM measurements. Dye doping was achieved by adding as low as $0.01 \%$ mol of dye with respect to TEOS. Furthermore, it could be easily adapted to other dyes, provided the possibility to their functionalization for the covalent bonding with the siloxane groups. This easy, time- and cost-efficient method to synthesise ultra-small fluorescent silica particles can open the way to the production of a new class of highly emitting luminescent markers for biosensing technologies. Finally, the procedure can be extended from a fluorescein-based dye to other fluorescent species for on-demand tuning of the optical properties.

Acknowledgements The authors wish to thank Diego Montagner (Maynooth University, Ireland) for NMR measurement of the FCPTES adduct, Piero Schiavuta (Protolab, Italy) for support in AFM measurements, Davide Cristofori (Università $\mathrm{Ca}^{\prime}$ Foscari Venezia, Italy) for TEM measurements, Filippo Romanato (Università di Padova, Italy) for high-resolution FESEM analysis, Alvise Benedetti (Università $\mathrm{Ca}^{\prime}$ Foscari Venezia, Italy), and Cinzia Sada (Università di Padova, Italy) for valuable discussions.

Funding information RR acknowledges the European Union's Horizon 2020 research and innovation programme under the $\mathrm{Ma}$ rie Sklodowska-Curie grant agreement no. 748649 (project "MNEMONIC"). FE acknowledges the PLESC project "Plasmonics for a better efficiency of solar cells" between South Africa and Italy ("Contributo del Ministero degli Affari Esteri e della Cooperazione Internazionale, Direzione Generale per la Promozione del Sistema Paese") and VINNOVA, the Swedish
Government Agency for Innovation, under the Vinnmer Marie Curie Incoming - Mobility for Growth Programme (project "Nano2solar" Ref. No. 2016-02011).

\section{Compliance with ethical standards}

Conflict of interest The authors declare that they have no conflict of interest.

Open Access This article is distributed under the terms of the Creative Commons Attribution 4.0 International License (http:// creativecommons.org/licenses/by/4.0/), which permits unrestricted use, distribution, and reproduction in any medium, provided you give appropriate credit to the original author(s) and the source, provide a link to the Creative Commons license, and indicate if changes were made.

\section{References}

Alemán JV, Chadwick AV, He J, Hess M, Horie K, Jones RG, Kratochvíl P, Meisel I, Mita I, Moad G, Penczek S, Stepto RFT (2007) Definitions of terms relating to the structure and processing of sols, gels, networks, and inorganic-organic hybrid materials (IUPAC Recommendations 2007). Pure Appl Chem 79:1801-1829

Asefa T, Zhimin T (2012) Biocompatibility of mesoporous silica nanoparticles. Review. Chem Res Toxicol 25(11):2265-2284

Bagwe RP, Yang C, Hilliard LR, Tan W (2004) Optimization of dye-doped silica nanoparticles prepared using a reverse microemulsion method. Langmuir 20:8336-8342

Banica FG (2012) Chemical sensors and biosensors: fundamentals and applications. John Wiley \& Sons, Chichester

Bogush GH, Tracy MA, Zukoski CF (1988) Preparation of monodisperse silica particles: control of size and mass fraction. $\mathrm{J}$ Non-Cryst Solids 1988(104):95-106

Brinker CJ, Scherer GW (1990) Sol-gel science: the physics and chemistry of sol-gel processing. Gulf Professional Publishing

Canton G, Riccò R, Marinello F, Carmignato S, Enrichi F (2011) Modified Stöber synthesis of highly luminescent dye-doped silica nanoparticles. J Nanopart Res 13:4349-4356

Cohen B, Martin C, Iyer SK, Wiesner U, Douhal A (2012) Single dye molecule behavior in fluorescent core-shell silica nanoparticles. Chem Mater 24:361-372

Enrichi F (2008) Luminescent amino-functionalized or erbiumdoped silica spheres for biological applications. Ann N Y Acad Sci 1130:262-266

Enrichi F, Riccò R, Parma A, Riello P, Benedetti A (2008) Synthesis and characterization of monodisperse Eu-doped luminescent silica nanospheres for biological applications. Proc SPIE 6991:69912E1-69912E8

Enrichi F, Riccò R, Scopece P, Parma A, Mazaheri AR, Riello P, Benedetti A (2009a) Comparison of Eu(NO3)3 and $\mathrm{Eu}(\mathrm{acac}) 3$ precursors for doping luminescent silica nanoparticles. J Nanopart Res 12:1925-1931

Enrichi F, Riccò R, Meneghello A, Pierobon R, Marinello F, Schiavuta P (2009b) Luminescent dye-doped or rare-earth- 
doped monodisperse silica nanospheres as efficient labels in DNA microarrays. Proc SPIE 7393:73930M

Enrichi F, Riccò R, Meneghello A, Pierobon R, Cretaio E, Marinello F, Schiavuta P, Parma A, Riello P, Benedetti A (2010) Investigation of luminescent dye-doped or rare-earthdoped monodisperse silica nanospheres for DNA microarray labelling. Opt Mater 32:1652-1658

Esquena J, Tadros TF, Kostarelos K, Solans C (1997) Preparation of narrow size distribution silica particles using microemulsions. Langmuir 13:6400-6406

Finnie KS, Bartlett JR, Barbé CJA, Kong L (2007) Formation of silica nanoparticles in microemulsions. Langmuir 23:30173024

Lade M, Mays H, Schmidt J, Willumeit R, Schomäcker R (2000) On the nanoparticle synthesis in microemulsions: detailed characterization of an applied reaction mixture. Colloids Surf Physicochem Eng Asp 163:3-15

Lakowicz JR (2006) Principles of fluorescent spectroscopy. Springer Verlag

Li Z, Barnes JC, Bosoy A, Stoddart JF, Zink JI (2012) Mesoporous silica nanoparticles in biomedical applications. Chem Soc Rev 41:2590-2605

Ma K, Sai H, Wiesner U (2012a) Ultrasmall sub-10 nm near IR fluorescent mesoporous silica nanoparticles. J Am Chem Soc 134:13180-13183
Ma K, Werner-Zwanziger U, Zwanziger J, Wiesner U (2012b) Controlling growth of ultrasmall sub-10 nm fluorescent mesoporous silica nanoparticles. Chem Mater 25:677-691

Neises B, Steglich W (1978) Simple method for the esterification of carboxylic acids. Angew Chem Int Ed Engl 17:522-524

Quan B, Lee C, Yoo JS, Wiesner U (2017) Facile scalable synthesis of highly monodisperse small silica nanoparticles using alkaline buffer solution and its application for efficient sentinel lymph node mapping. J Mater Chem B 5:586-594

Riccò R, Meneghello A, Enrichi F (2011) Signal enhancement in DNA microarray using dye doped silica nanoparticles: application to human papilloma virus (HPV) detection. Biosens Bioelectron 26:2761-2765

Stöber W, Fink A, Bohn E (1968) Controlled growth of monodisperse silica spheres in the micron size range. J Colloid Interface Sci 26:62-69

Syahir A, Usui K, Tomizaki K, Kajikawa K, Mihara H (2015) Label and label-free detection techniques for protein microarrays. Microarrays 4:228-244

van Blaaderen A, Vrij A (1992) Synthesis and characterization of colloidal dispersions of fluorescent, monodisperse silica spheres. Langmuir 8:2921-2931 